\title{
Circular edge-colorings of cubic graphs with girth six
}

\author{
Daniel Král* $\quad$ Edita Máčajová ${ }^{\dagger} \quad$ Ján Mazák ${ }^{\ddagger}$ \\ Jean-Sébastien Sereni ${ }^{\S}$
}

\begin{abstract}
We show that the circular chromatic index of a (sub)cubic graph with odd-girth at least 7 is at most $7 / 2$.
\end{abstract}

\section{Introduction}

A classical theorem of Vizing [14] asserts that the chromatic index of every cubic bridgeless graph, i.e., the smallest number of colors needed to properly edge-color such a graph, is 3 or 4. Cubic cyclically 4-edge-connected graphs

${ }^{*}$ Institute for Theoretical Computer Science (ITI), Faculty of Mathematics and Physics, Charles University, Malostranské náměstí 25, 11800 Prague 1, Czech Republic. This research was partially supported by the grant GACR 201/09/0197. E-mail: kral@kam.mff.cuni.cz. Institute for Theoretical Computer Science is supported as project 1M0545 by Czech Ministry of Education.

${ }^{\dagger}$ Department of Computer Science, Faculty of Mathematics, Physics and Informatics, Comenius University, Mlynská dolina, 84248 Bratislava, Slovakia. This author's work was partially supported by the APVV project 0111-07. E-mail: macajova@dcs.fmph.uniba.sk.

${ }^{\ddagger}$ Department of Computer Science, Faculty of Mathematics, Physics and Informatics, Comenius University, Mlynská dolina, 84248 Bratislava, Slovakia. This author's work was partially supported by the grant UK/384/2009 and by the APVV project 0111-07. E-mail: mazak@dcs.fmph.uniba.sk.

${ }^{\S}$ CNRS (LIAFA, Université Denis Diderot), Paris, France, and Department of Applied Mathematics (KAM) - Faculty of Mathematics and Physics, Charles University, Prague, Czech Republic. This author's work was partially supported by the Égide ECO-NET project 16305SB. E-mail: sereni@kam.mff.cuni.cz. 
with chromatic index 4 are known as snarks and it is known that smallest counter-examples, if any, to several deep open conjectures in graph theory, such as the Cycle Double Cover Conjecture, must be snarks.

Our research is motivated by edge-colorings of cubic bridgeless graphs with no short cycles. The Girth Conjecture of Jaeger and Swart [5] asserted that there are no snarks with large girth. This conjecture was refuted by Kochol [8] who constructed snarks of arbitrary large girth. Hence, it is natural to ask whether it can be said that snarks of large girth are close to being 3 -edge-colorable in some sense.

One of the relaxations of ordinary colorings are circular colorings, introduced by Vince [13]. A $(p, q)$-coloring of a graph $G$ is a coloring of vertices with colors from the set $\{1, \ldots, p\}$ such that any two adjacent vertices receive colors $a$ and $b$ with $q \leq|a-b| \leq p-q$. Circular colorings naturally appear in different settings, which is witnessed by several equivalent definitions of this notion as exposed in the surveys by Zhu $[15,16]$.

The infimum of the ratios $p / q$ such that $G$ has a $(p, q)$-coloring is the circular chromatic number of $G$. It is known that the infimum is the minimum for all finite graphs and the ceiling of the circular chromatic number of a graph is equal to its chromatic number. Thus, the circular chromatic number is a fractional relaxation of the chromatic number. The circular chromatic index of a graph is the circular chromatic number of its line-graph.

Zhu [15] asked whether there exist snarks with circular chromatic index close or equal to 4 , and as there are snarks with arbitrary large girth, it is also interesting to know whether there exist such snarks of arbitrary large girth. Afshani et al. [1] showed that the circular chromatic index of every cubic bridgeless graph is at most $11 / 3$ and Kaiser et al. [6] showed that for every $\varepsilon>0$, there exists $g$ such that every cubic bridgeless graph with girth at least $g$ has circular chromatic index at most $3+\varepsilon$. This latter result was generalized to graphs with bounded maximum degree [7]. Moreover, the circular chromatic indices of several well-known classes of snarks have been determined $[2,3,4,10]$.

The Petersen graph is the only cubic bridgeless graph that is known to have the circular chromatic index equal to $11 / 3$. In fact, it is the only example of a cubic bridgeless graph with circular chromatic index greater than $7 / 2$. This leads to the following (wild) conjecture.

Conjecture 1. Every cubic bridgeless graph different from the Petersen graph has circular chromatic index strictly less than 11/3, maybe, at most 
$7 / 2$

In their paper, Kaiser et al. [6], formulated a problem to determine the smallest girth $g_{0}$ such that every cubic bridgeless graph with girth at least $g_{0}$ has circular chromatic index at most $7 / 2$, and they showed that $g_{0} \leq 14$. Note that $g_{0} \geq 6$ because of the Petersen graph. In this paper, we prove that every cubic bridgeless graph having a 2-factor composed of cycles of length different from 3 and 5 has circular chromatic index at most $7 / 2$. This implies that $g_{0}=6$, i.e., the circular chromatic index of every cubic bridgeless graph with girth at least 6 is at most $7 / 2$. Our result also applies to subcubic graphs with odd-girth at least 7 .

\section{Compatible trails}

The core of our argument is formed by decomposing the graph obtained by contracting a 2-factor of a cubic bridgeless graph into trails. We first introduce notation related to such decompositions and then prove their existence.

\subsection{Notation}

An abstract map is a graph with multiple edges, loops and half-edges allowed with a fixed cyclic ordering of the ends around each vertex. Formally, an abstract map $(V, E, \varphi)$ is comprised of a vertex-set $V$ and an edge-set $E$. Each edge has two ends: one of them is incident with a vertex, and the other may, but need not, be incident with a vertex. An edge that has exactly one end incident with a vertex is a half-edge. An edge with both ends incident with the same vertex is a loop. The degree $d_{v}$ of a vertex $v \in V$ is the number of ends incident with $v$ (in particular, loops are counted twice). Moreover, for each vertex, there is a cyclic ordering of the ends incident with it. These orderings are represented by a surjective mapping $\varphi: V \times \mathbb{N} \rightarrow E$ such that $\varphi(v, n)$ is an edge incident with $v$ and $\varphi(v, n)=\varphi\left(v, n+d_{v}\right)$ for every $(v, n) \in V \times \mathbb{N}$. An edge has two pre-images in $\{v\} \times\left\{1, \ldots, d_{v}\right\}$ if and only if it is a loop incident with $v$. An abstract map naturally yields an embedding of the corresponding multi-graph on a surface.

Deleting a vertex $v \in V$ from an abstract map $G=(V, E, \varphi)$ yields an abstract map $G^{\prime}=\left(V^{\prime}, E^{\prime}, \varphi^{\prime}\right)$ with $V^{\prime}=V \backslash\{v\}, E^{\prime}=\varphi\left(V^{\prime} \times \mathbb{N}\right)$ and $\varphi^{\prime}$ being the restriction of $\varphi$ to $V^{\prime} \times \mathbb{N}$. In other words, the vertex $v$ and half- 
edges and loops incident with $v$ are removed. The other edges incident with $v$ become half-edges.

A trail in an abstract map is a sequence of mutually distinct edges $e_{1}, e_{2}, \ldots, e_{k}$ such that

- $e_{i}$ and $e_{i+1}$ have a common end-vertex $v_{i}$, for $i \in\{1, \ldots, k-1\}$; and

- $v_{i-1} \neq v_{i}$ unless $e_{i}$ is a loop, for $i \in\{2, \ldots, k-1\}$.

If $e_{1}$ is not a half-edge, then the trail starts at a vertex. Similarly, if $e_{k}$ is not a half-edge, the trail ends at a vertex. Furthermore, a trail that consists of a single half-edge either starts or ends at a vertex. For a given trail, a linear ordering on its edges is naturally defined. Let $T_{1}$ and $T_{2}$ be two edge-disjoint trails and $v$ a vertex. If $T_{1}$ ends at $v$ and $T_{2}$ starts at $v$, then we can link the two trails by identifying the end of $T_{1}$ with the beginning of $T_{2}$ : thereby, we obtain a new trail that first follows the edges of $T_{1}$ and then those of $T_{2}$. We also define the linking of a trail $T$ that starts and ends at the same vertex with itself: in this case, we obtain the same trail $T$ except that the ordering of the edges becomes cyclic. A trail obtained in this way is closed. Trails that are not closed are open. Note that a trail that starts and ends at the same vertex can be either open or closed.

Trails $W_{1}, \ldots, W_{K}$ form a compatible decomposition of an abstract map $G$ if all of them are open, every edge is contained in exactly one of the trails and for every vertex $v$ of odd degree of $G$, there is an index $i_{v}$ such that the following pairs of edges are consecutive (regardless of their order) in some of the trails (and thus are not the same edge):

- $\varphi\left(v, i_{v}\right)$ and $\varphi\left(v, i_{v}+5\right)$;

- $\varphi\left(v, i_{v}+1\right)$ and $\varphi\left(v, i_{v}+3\right)$; and

- $\varphi\left(v, i_{v}+2\right)$ and $\varphi\left(v, i_{v}+4\right)$.

Note that if an abstract map $G$ has a compatible decomposition, then it has no vertices of degree 1 or 5 .

\section{$2.2 \quad$ Existence}

We now prove that every abstract map with no vertices of degree 1, 3 or 5 has a compatible decomposition. In the next two lemmas, which form the base of our inductive argument, abstract maps with a single vertex are analyzed. 


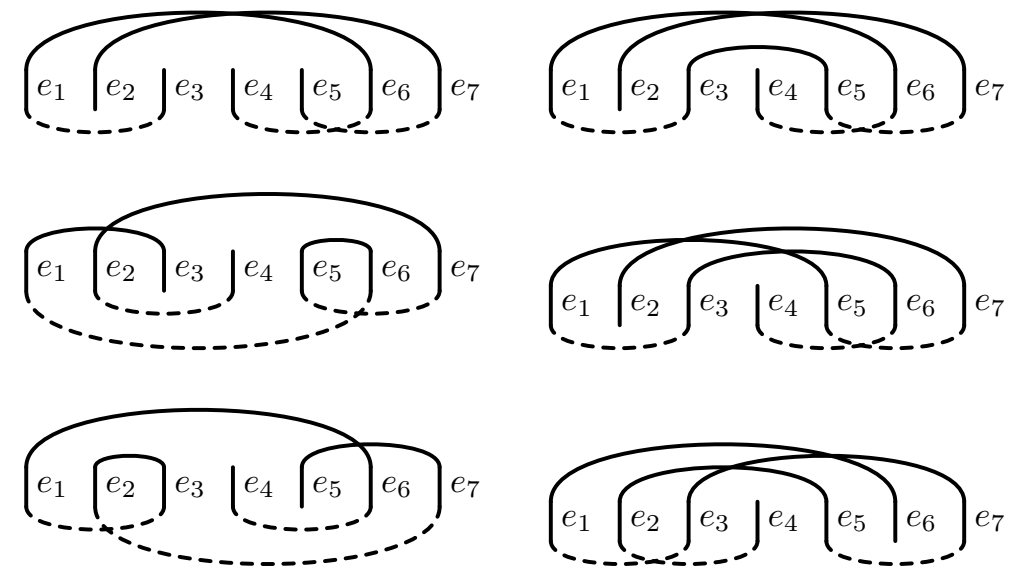

Figure 1: Compatible decompositions in the first (the top two pictures) and the last four (the remaining pictures) main cases in the proof of Lemma 1. The edges incident with the vertex $v$ are drawn with solid lines and the way in which they are joined to form the trails of the decomposition is indicated by dashed lines.

Lemma 1. Every abstract map $G$ that has a single vertex and the degree of this vertex is 7 has a compatible decomposition.

Proof. Let $v$ be the vertex and let $e_{i}$ be the image of $\varphi(v, i)$ for each $i \in$ $\{1,2, \ldots, 7\}$. Further, let $\mathcal{W}_{i}$ be the set of trails obtained by the following process: initially, each edge of $G$ forms a single trail. We then link the two trails starting or ending with $e_{i}$ and $e_{i+5}$, next we link the two trails starting or ending with $e_{i+1}$ and $e_{i+3}$, and last the two trails starting or ending with $e_{i+2}$ and $e_{i+4}$. This operation does not always yield a compatible decomposition, since it may create closed trails. For instance, $\mathcal{W}_{i}$ contains a closed trail if $e_{i}=e_{i+5}$, or if $e_{i+1}=e_{i+4}$ and $e_{i+2}=e_{i+3}$ (in this last case, after having linked $e_{i+1}$ with $e_{i+3}$, thereby obtaining a trail $T$, the last linking amounts to linking $T$ with itself). On the other hand, if all the obtained trails are open, then $\mathcal{W}_{i}$ is a compatible decomposition, with $i$ being the index $i_{v}$ of the definition.

Our goal is to show that at least one of the sets $\mathcal{W}_{i}$ is composed only of open trails. Since the degree of $v$ is odd, the vertex $v$ is incident with at least one half-edge. By symmetry, we assume that this half-edge is $e_{4}$.

If $\mathcal{W}_{1}$ contains a closed trail, then one of the following four cases applies 
(recall that $e_{4}$ is a half-edge):

- $e_{1}=e_{6}$,

- $e_{3}=e_{5}$,

- $e_{1}=e_{3}$ and $e_{5}=e_{6}$, or

- $e_{1}=e_{5}$ and $e_{3}=e_{6}$.

Similarly, if $\mathcal{W}_{2}$ contains a closed trail, then one of the following four cases applies:

- $e_{2}=e_{7}$,

- $e_{3}=e_{5}$,

- $e_{2}=e_{3}$ and $e_{5}=e_{7}$, or

- $e_{2}=e_{5}$ and $e_{3}=e_{7}$.

Comparing the two sets of four possible cases, we conclude that if none of $\mathcal{W}_{1}$ and $\mathcal{W}_{2}$ is compatible, then at least one of the following six cases applies (these cases are referred to as main cases in Figures 1 and 2):

- $e_{1}=e_{6}$ and $e_{2}=e_{7}$,

- $e_{3}=e_{5}$,

- $e_{1}=e_{3}, e_{5}=e_{6}$ and $e_{2}=e_{7}$,

- $e_{1}=e_{5}, e_{3}=e_{6}$ and $e_{2}=e_{7}$,

- $e_{1}=e_{6}, e_{2}=e_{3}$ and $e_{5}=e_{7}$, or

- $e_{1}=e_{6}, e_{2}=e_{5}$ and $e_{3}=e_{7}$.

In the first case, the set $\mathcal{W}_{3}$ contains only open trails. In the last four cases, the sets $\mathcal{W}_{4}, \mathcal{W}_{3}, \mathcal{W}_{6}$ and $\mathcal{W}_{7}$, respectively, are composed of open trails (see Figure 1 for an illustration).

We now focus on the second case. If $\mathcal{W}_{3}$ contains a closed trail (and $\left.e_{3}=e_{5}\right)$, it holds that $e_{1}=e_{7}$. However, in this case, the set $\mathcal{W}_{4}$ contains no closed trails (see Figure 2). This finishes the proof of the lemma. 

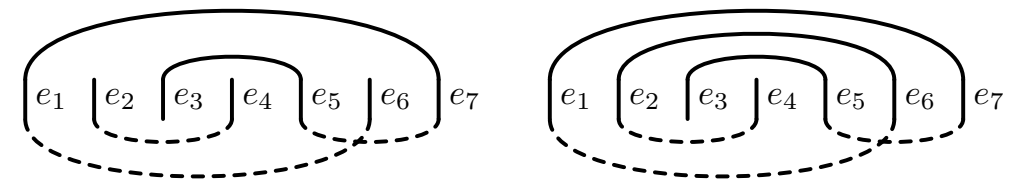

Figure 2: Compatible decompositions in the second main case in the proof of Lemma 1.

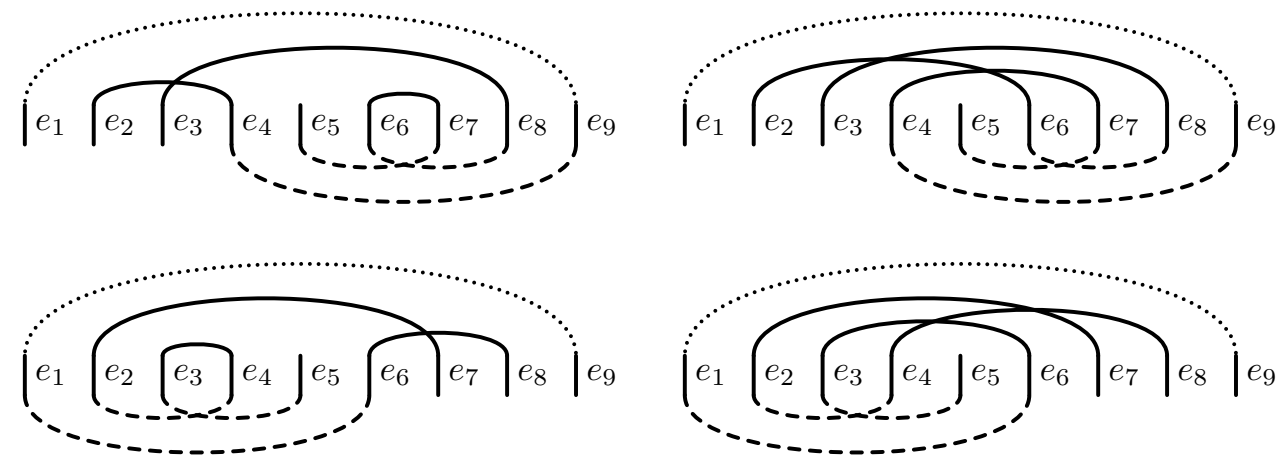

Figure 3: Compatible decompositions in the last four cases in the proof of Lemma 2. Additional loops that can also be present are drawn with dotted lines.

Lemma 2. Every abstract map $G$ that has a single vertex and the degree of this vertex is different from 1,3 and 5 has a compatible decomposition.

Proof. Let $v$ be the only vertex of the graph $G$. If the degree of $v$ is even, then there is nothing to prove as every decomposition into open trails is compatible. If the degree of $v$ is 7 , then the statement follows from Lemma 1. Hence, we assume that the degree of $v$ is odd and it is at least 9 . Let $e_{i}$ be the image of $\varphi(v, i)$ for $i \in\{1,2, \ldots, 9\}$.

As the degree of $v$ is odd, $v$ is incident with at least one half-edge. Without loss of generality, we can assume that $e_{5}$ is the half-edge. Let $\mathcal{W}_{i}$ be the set of trails defined as in the proof of Lemma 1. Assume that both the sets $\mathcal{W}_{2}$ and $\mathcal{W}_{3}$ contain a closed trail; if any of them were composed of open trails only, then it would form a compatible decomposition.

As in the proof of Lemma 1, we infer from the facts that both $\mathcal{W}_{2}$ and $\mathcal{W}_{3}$ contain a closed trail that one of the following six cases applies (replace $e_{4}$ with $e_{5}$ and $\mathcal{W}_{i}$ with $\mathcal{W}_{i+1}$ for $i \in\{1,2\}$ in the analysis done in the proof 

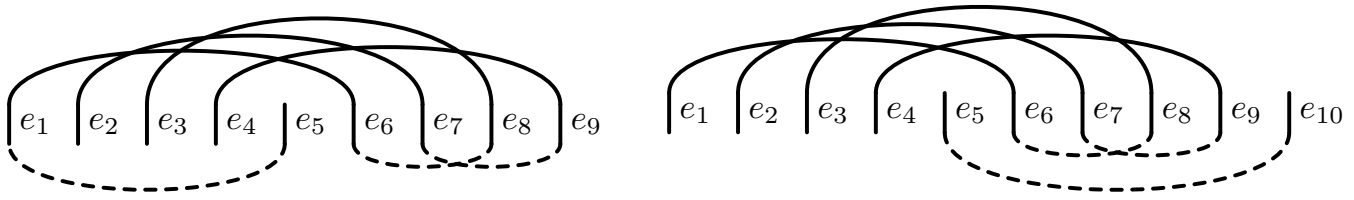

Figure 4: Compatible decompositions in the case where $e_{2}=e_{7}$ and $e_{3}=e_{8}$ in the proof of Lemma 2. Two cases are distinguished based on whether the degree of $v$ is equal to 9 or not.

of Lemma 1):

- $e_{2}=e_{7}$ and $e_{3}=e_{8}$,

- $e_{4}=e_{6}$,

- $e_{2}=e_{4}, e_{6}=e_{7}$ and $e_{3}=e_{8}$,

- $e_{2}=e_{6}, e_{4}=e_{7}$ and $e_{3}=e_{8}$,

- $e_{2}=e_{7}, e_{3}=e_{4}$ and $e_{6}=e_{8}$, or

- $e_{2}=e_{7}, e_{3}=e_{6}$ and $e_{4}=e_{8}$.

In the last four cases, the sets $\mathcal{W}_{4}, \mathcal{W}_{4}, \mathcal{W}_{1}$ and $\mathcal{W}_{1}$, respectively, contain no closed trails (see Figure 3 for an illustration). Let us focus on the first two cases, now.

Suppose that $e_{2}=e_{7}$ and $e_{3}=e_{8}$. If $\mathcal{W}_{1}$ contains a closed trail, then $e_{1}=e_{6}$. Further, if $\mathcal{W}_{4}$ contains a closed trail, then $e_{4}=e_{9}$. Thus, the set $\mathcal{W}_{5}$ contains no closed trails (see Figure 4 ), and hence is a compatible decomposition.

Assume now that $e_{4}=e_{6}$. If $\mathcal{W}_{1}$ contains a closed trail, then $e_{1}=e_{2}$. Further, if $\mathcal{W}_{4}$ contains a closed trail, then $e_{8}=e_{9}$. Again, the set $\mathcal{W}_{5}$ is then a compatible decomposition (see Figure 5).

To summarize, we have shown that at least one of the sets $\mathcal{W}_{i}, i \in$ $\{1,2,3,4,5\}$, is composed of open trails only, and thus it forms a compatible decomposition.

Using Lemma 2, we show that every abstract map with no vertices of degree 1,3 or 5 has a compatible decomposition. 


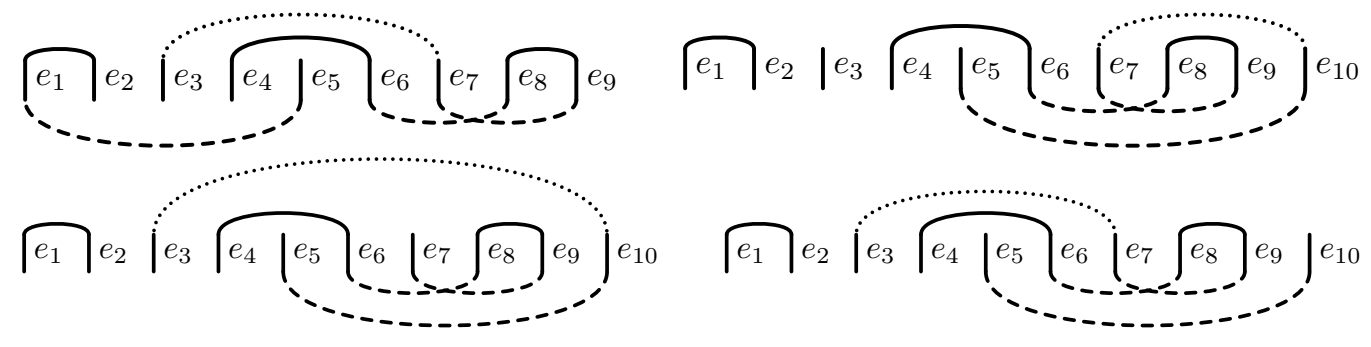

Figure 5: Compatible decompositions in the case where $e_{4}=e_{6}$ in the proof of Lemma 2. Two cases are distinguished based on whether the degree of $v$ is equal to 9 or not. Additional loops that can also be present are drawn with dotted lines.

Lemma 3. Every abstract map $G$ without vertices of degree 1, 3 or 5 has a compatible decomposition.

Proof. The proof proceeds by induction on the number of vertices of $G$. If $G$ has one vertex, then the statement follows from Lemma 2. Otherwise, let $v$ be an arbitrary vertex of $G$ and let $G^{\prime}$ be an abstract map obtained from $G$ by removing $v$. By the induction hypothesis, $G^{\prime}$ has a compatible decomposition $\mathcal{W}^{\prime}$ into trails.

Let $\mathcal{W}$ be the set of trails obtained from $\mathcal{W}^{\prime}$ by adding the set of loops and half-edges incident with $v$ in $G$. Observe that $\mathcal{W}$ is a set of trails of $G$ in which there is no trail "traversing" $v$. If the degree of $v$ is even, the set $\mathcal{W}$ is a compatible decomposition of $G$ as there is no restriction on how the trails pass through the vertex $v$. Assume that the degree $d_{v}$ of $v$ is odd.

We now define an auxiliary abstract map $H$. The abstract map $H$ contains a single vertex $w$ of degree $d_{v}$, and for $(i, j) \in\left\{1,2, \ldots, d_{v}\right\}^{2}$, we have $\varphi(w, i)=\varphi(w, j)$ if and only if $\mathcal{W}$ contains a trail starting with the edge $\varphi(v, i)$ and ending with $\varphi(v, j)$. In other words, trails of $\mathcal{W}^{\prime}$ that both start and finish with an edge incident with $v$ correspond in $H$ to loops incident with $w$, the loops incident with $v$ are preserved and the trails starting or finishing at $v$ (but not both) correspond to half-edges. Trails containing no edge incident with $v$ have no counterparts among the edges of $H$.

By Lemma 2, the abstract map $H$ has a compatible decomposition $\mathcal{W}_{H}$. We can now obtain a compatible decomposition $\mathcal{W}_{G}$ of $G$ as follows: all the trails of $\mathcal{W}$ neither starting nor ending at $v$ are added to $\mathcal{W}_{G}$. Each trail $W$ of $\mathcal{W}_{H}$ has a corresponding trail in $\mathcal{W}_{G}$ that is obtained by replacing every 
edge of $W$ with the corresponding trail of $\mathcal{W}$ and linking these trails.

It is straightforward to verify that $\mathcal{W}_{G}$ is a set of open trails of $G$. By induction, the trails pass through vertices of $G$ different from $v$ in the way required by the definition of a compatible decomposition. The trails also pass through $v$ in the required way because $\mathcal{W}_{H}$ is a compatible decomposition of $H$. Hence, $\mathcal{W}_{G}$ is a compatible decomposition of $G$.

\section{$3 \quad$ Graphs with odd-girth at least 7}

We are now ready to prove our main theorem.

Theorem 4. The circular chromatic index of every cubic graph with a 2factor composed of cycles of lengths different from 3 and 5 is at most $7 / 2$.

Proof. Let $G$ be a cubic graph and $F$ a 2-factor of $G$ composed of cycles of lengths different from 3 and 5 , and let $M$ be the perfect matching complementary to $F$. The multi-graph obtained by contracting $F$ can be viewed as an abstract map $H$ : the vertices of $H$ correspond to the cycles of the 2 -factor $F$, and the order in which the edges of $M$ are incident with cycles of $F$ naturally defines the function $\varphi$. Note that $H$ has no half-edges and its loops correspond to chords of cycles of $F$.

Since no cycle of $F$ has length 3 or 5 , no vertex of $H$ has degree 1,3 or 5 . Thus, by Lemma 3 , the abstract map $H$ has a compatible decomposition $\mathcal{W}$. Color the edges of every trail of $\mathcal{W}$ with 0 and 1 in an alternating way. Since the edges of $H$ correspond one-to-one to the edges of $M$, we have obtained a coloring of the edges of $M$ with 0 and 1.

We now construct a $(7,2)$-edge-coloring of the edges of $G$. Let $C=$ $v_{0} v_{1} \cdots v_{\ell-1}$ be a cycle of $F$ and $c_{i}$ the color of the edge of $M$ incident with the vertex $v_{i}$, for $i \in\{0,1, \ldots, \ell-1\}$. If the length $\ell$ of $C$ is even, we color the edges of $C$ with 3 and 5 in an alternating way. Let us consider the case where $\ell$ is odd. Since $\mathcal{W}$ is a compatible decomposition, there exists an index $k$ such that $c_{k} \neq c_{k+5}, c_{k+1} \neq c_{k+3}$ and $c_{k+2} \neq c_{k+4}$ (indices are taken modulo $\ell)$ as the colors of the edges of trails of $\mathcal{W}$ alternate.

We now show that there exists an index $k^{\prime}$ such that $c_{k^{\prime}}=c_{k^{\prime}+1} \neq c_{k^{\prime}+2}=$ $c_{k^{\prime}+3}$. If $c_{k+1}=c_{k+2}$, then set $k^{\prime}=k+1$. Otherwise, $c_{k+1}=c_{k+4} \neq c_{k+2}=$ $c_{k+3}$. Since either $c_{k}$ or $c_{k+5}$ is equal to $c_{k+1}=c_{k+4}$, the index $k^{\prime}$ can be set to $k$ or $k+2$. 


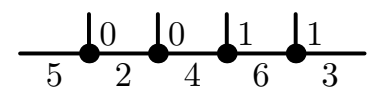

Figure 6: Coloring odd cycles in the proof of Theorem 4.

By symmetry, we can assume in the remainder that $k^{\prime}=1, c_{1}=c_{2}=0$ and $c_{3}=c_{4}=1$. Color the edge $v_{1} v_{2}$ with 2 , the edge $v_{2} v_{3}$ with 4 and the edge $v_{3} v_{4}$ with 6 . The remaining edges are colored with 3 and 5 in the alternating way (see Figure 6). We have obtained a proper coloring of $C$. As we can extend the coloring of the edges of $M$ to all cycles of $F$, the resulting $(7,2)$-edge-coloring witnesses that the circular chromatic index of $G$ does not exceed $7 / 2$.

Petersen's theorem [11] asserts that every cubic bridgeless graph has a perfect matching; this yields the next corollary of Theorem 4.

Corollary 5. The circular chromatic index of every cubic bridgeless graph with girth 6 or more is at most $7 / 2$.

Finally, we show that the assumption that the given graph is cubic can be relaxed in Corollary 5 .

Corollary 6. The circular chromatic index of every subcubic graph with oddgirth 7 or more is at most $7 / 2$.

Proof. Let $G$ be a subcubic graph with odd-girth 7 or more that has a circular chromatic index greater than $7 / 2$ and that has the smallest number of vertices among all such graphs. Consequently, the minimum degree of $G$ is at least 2. Similarly, $G$ is connected. The graph $G$ is also bridgeless: otherwise, each of the two graphs obtained from $G$ by splitting along the bridge has a $(7,2)$ edge-coloring and these edge-colorings (after rotating the colors if necessary) combine to a $(7,2)$-edge-coloring of $G$.

If $G$ has no vertices of degree 2 , then $G$ is a cubic bridgeless graph. Petersen's theorem [11] ensures that $G$ has a a 2 -factor $F$. By our assumption, no cycle of $F$ has length 3 or 5 , and therefore $G$ cannot be a counter-example by Theorem 4 . So, assume that $G$ has at least one vertex of degree 2 . Let $H$ be a 3-edge-connected cubic graph of odd-girth at least 7 from which we remove an edge. We construct the graph $G^{\prime}$ as follows. We take two disjoint copies of $G$. For each pair $(u, v)$ of corresponding vertices of degree 2 (one 
in each copy of $G$ ), we add a copy of $H$, join $u$ to a vertex of degree 2 of $H$, and $v$ to the other vertex of degree 2 in $H$. The resulting graph $G^{\prime}$ is cubic and has odd-girth at least 7. Moreover, since $G$ is bridgeless, $G^{\prime}$ has at most two bridges. More precisely, $G^{\prime}$ has two bridges if and only if $G$ has exactly one vertex of degree 2 , and $G^{\prime}$ is bridgeless otherwise. Therefore, $G^{\prime}$ has a perfect matching by Tutte's theorem $[9,12]$. Consequently, since $G^{\prime}$ has odd-girth at least 7, Theorem 4 implies the existence of a (7,2)-edge-coloring of $G^{\prime}$. This edge-coloring restricted to $G$ yields a $(7,2)$-edge-coloring of $G$, a contradiction.

\section{Acknowledgment}

The first and the last authors would like to thank Mohammad Ghebleh and Luke Postle for discussions and insights on circular edge-colorings of cubic bridgeless graphs.

\section{References}

[1] P. Afshani, M. Ghandehari, M. Ghandehari, H. Hatami, R. Tusserkani, $\mathrm{X}$. Zhu: Circular chromatic index of graphs of maximum degree 3, J. Graph Theory 49 (2005), 325-335.

[2] M. Ghebleh, D. Král', S. Norine, R. Thomas: The circular chromatic index of flower snarks, Electron. J. Combin. 13 (2006), \#N20, 7pp.

[3] M. Ghebleh: The circular chromatic index of Goldberg snarks, Discrete Math. 307 (2007), 3220-3225.

[4] M. Ghebleh: The circular chromatic index of generalized Blanuša snarks, Electron. J. Combin. 15 (2008), \#R44, 9pp.

[5] F. Jaeger, T. Swart: Conjecture 1, in: Combinatorics 79 (M. Deza, I. G. Rosenberg, eds.), Ann. Discrete Math. Vol. 9, North-Holland, Amsterdam, 1980, p. 305.

[6] T. Kaiser, D. Král', R. Škrekovski: A revival of the girth conjecture, J. Combin. Theory Ser. B 92 (2004), 41-53. 
[7] T. Kaiser, D. Král', R. Škrekovski, X. Zhu: The circular chromatic index of graphs of high girth, J. Combin. Theory Ser. B 97 (2007), 1-13.

[8] M. Kochol: Snarks without small cycles, J. Combin. Theory Ser. B 67 (1996), 34-47.

[9] L. Lovász, M. D. Plummer: Matching theory, Volume 121 of NorthHolland Mathematics Studies. North-Holland Publishing Co., Amsterdam, 1986. Annals of Discrete Mathematics, 29.

[10] J. Mazák: Circular chromatic index of type 1 Blanuša snarks, J. Graph Theory 59 (2008), 89-96.

[11] J. Petersen: Die Theorie der regulären graphs, Acta Math. 15 (1891), 193-220.

[12] W. T. Tutte: The Factorization of Linear Graphs, J. London Math. Soc. 22 (1947), 107-111.

[13] A. Vince: Star chromatic number, J. Graph Theory 12 (1988), 551-559.

[14] V. G. Vizing: On an estimate of the chromatic class of a $p$-graph (in Russian), Diskret. Analiz. 3 (1964), 24-30.

[15] X. Zhu: Circular chromatic number: a survey, Discrete Math. 229 (2001), 371-410.

[16] X. Zhu: Recent developments in circular colorings of graphs, in M. Klazar, J. Kratochvíl, J. Matoušek, R. Thomas, P. Valtr (eds.): Topics in Discrete Mathematics, Springer, 2006, 497-550. 